\title{
Epicardial Posterior Papillary Muscle Repositioning with Mitral Annular Reduction for FIMR Treatment: Initial Ex Vivo Heart Model Study
}

\author{
Krishaporn Kradangnga, DVM, $\mathbf{P h D}^{1,2}$ Eric Monnet, DVM, $\mathrm{PhD}^{1}$ \\ ${ }^{1}$ Department of Clinical Sciences, College of Veterinary Medicine and Biomedical Sciences, Colorado State University, Fort Collins, \\ Colorado, USA; ${ }^{2}$ Department of Veterinary Surgery, Faculty of Veterinary Science, Chulalongkorn University, Bangkok, Thailand
}

\section{ABSTRACT}

Background: High recurrent functional ischemic mitral regurgitation (FIMR) has been observed after annuloplasty. Since annuloplasty alone could not prevent late recurrent FIMR or improve the survival rate after CABG, adjunctive subvalvular opt for better treatment tailored for each individual patient.

Methods: Ex vivo ovine heart models with annular dilatation and PPM displacement were used for analysis of mitral regurgitation (MR) flow, left ventricular and annular geometry after treatment by mitral annular reduction alone (MA, $\mathrm{n}_{\mathrm{MA}}=12$ ) or combined with epicardial PPM repositioning (MA+PPM, $\mathrm{n}_{\mathrm{MA}+\mathrm{PPM}}=13$ ).

Results: MR significantly was reduced from baseline in both the MA $(P=.03)$ and MA+PPM $(P=.02)$ groups, but was not significantly different between the groups. The septo-lateral mitral annular distance decreased after applying both methods (MA group $P=.005$; MA+PPM group $P=.05$ ). The tethering $\alpha$ angle of the APM in the frontal plane significantly increased from baseline in the MA+PPM group $(P$ $=.027)$. Furthermore, the MA+PPM group had a larger APM and PPM $\alpha$ angle in the frontal plane compared with the MA group after reducing the $\mathrm{MR}(P=.04)$. There were no statistically significant changes in tethering angles found in the MA group compared with baseline. MR reduction correlated with percentage decrease of septo-lateral mitral annular distance $\left(r_{s}=0.51, P=.01\right)$, the percentage decrease of fibrosa-PPM distance $\left(\mathrm{r}_{\mathrm{s}}=0.43, P=.03\right)$, and the percentage increase of the PPM anterior displacement $\left(r_{s}=-0.41, P=.04\right)$.

Conclusion: The decreased tethered angle of the PPM referred to the annulus, and the decreased interpapillary muscles distance suggested the PPM was repositioned inward and toward the septal annulus by the epicardial pushing pad. Epicardial repositioning of the PPM adjunct with mitral annular reduction facilitated leaflet coaptation without the risk of overlying restriction of the mitral annular orifice.

Received September 1, 2019; accepted October 13, 2019

Correspondence: Krishaporn Kradangnga, Department of Clinical Sciences, College of Veterinary Medicine and Biomedical Sciences, Colorado State University, Fort Collins, Colorado, USA (e-mail: krishaporn@gmail.com).

\section{INTRODUCTION}

Functional ischemic mitral regurgitation is associated with complications and poor prognosis after cardiac surgery [Badiwala 2009; Kumanohoso 2003]. Despite mild mitral regurgitation, the risk of heart failure and mortality significantly increases compared with normal patients, and the outcomes worsen with increased severity of FIMR [Bursi 2005; Fattouch 2010]. Regarding normal mitral leaflet structures, FIMR is related to dilatation of the mitral annulus and restriction of the mitral leaflets during systole due to PMs displacement.

FIMR is widely accepted as a "ventricular" problem [Bolling 2001]. Papillary muscles displacement induces mitral valve tethering, which results in mitral regurgitation from the loss of normal coaptation of the leaflets. In addition to $\mathrm{CABG}$, ring annuloplasty with or without reduction (or restricted mitral annuloplasty) is currently used as the method of choice to treat FIMR [Members, 2006 Writing Committee 2008; Members AF 2012; Members WC 2011]. The concept of ring annuloplasty is to bring the annular dimension closer together so the mitral leaflets could completely form coaptation. This technique has led to a high success rate in

Table 1. Piezo-Electric Crystal Pairs Used for Tethered Distance Measurements and Reference Markers for Cartesian Plane Construction

\begin{tabular}{lc}
\hline Crystal pair & Distance between mitral apparatus landmark \\
\hline 1 and 2 & Septo-lateral diameter of the annulus/Lateral axis/Minor axis I \\
1 and 3 & Anterior axis \\
1 and 4 & Apical axis \\
1 and 5 & Fibrosa-APM tip distance \\
1 and 6 & Fibrosa-PPM tip distance \\
2 and 5 & Lateral annulus to APM tip distance \\
2 and 6 & Lateral annulus to PPM tip distance \\
3 and 4 & Major axis \\
5 and 6 & Interpapillary muscle distance/Minor axis II
\end{tabular}

Abbreviations: APM, anterior papillary muscle; PPM, anterior papillary muscle 
Table 2. Data of the MR severity, MAA, and LVV

\begin{tabular}{lccccc}
\hline & \multicolumn{2}{c}{ MA Group $(\mathrm{N}=12)$} & \multicolumn{2}{c}{ MA+PPM Group $(\mathrm{N}=13)$} \\
PPM tip & Baseline & Treatment & Baseline & Treatment \\
\hline MR grade & $1[1,3]$ & $1 *[0.5,1]$ & $1[1,2]$ & $1 *[0,1]$ & .75 \\
MR reduction $(\%)$ & 0 & $75.61 *[39.71,100]$ & 0 & $70.0 *[60.0,100.0]$ & .50 \\
MAA $\left(\mathrm{cm}^{2}\right)$ & $8.47[5.06,15.66]$ & $6.22 *[3.76,9.35]$ & $8.79[5.51,12.89]$ & $11.24[6.08,11.68]$ & .14 \\
$\operatorname{LVV}(\mathrm{mL})$ & $42.79[25.95,59.06]$ & $27.43 *[22.65,34.76]$ & $35.61[33.09,41.7]$ & $32.93 *[25.83,38.54]$ & .26 \\
\hline
\end{tabular}

Values are in medians and interquartile ranges. MR, mitral regurgitation; MAA, mitral annular area; LVV, left ventricular volume. *Denotes $P<.05$ versus Baseline. †Relative change (\%) after epicardial device placement in the MA+PPM versus MA group.

short-term studies [Bolling 2001; Daneshmand 2009; Otsuji 2008]. Despite early satisfactory outcomes of this technique, late recurrence of FIMR has been observed in a significant number of cases [Calafiore 2001; Gillinov 2001; Jensen 2010; McGee 2004]. Post annuloplasty progression of left ventricular (LV) remodeling and mitral valve tethering could impair outcomes from annuloplasty alone [Hung 2004]. Adjunct procedures should be used to address the LV remodeling and prevent recurrence of FIMR after surgery.

Since annuloplasty alone could not prevent late recurrent FIMR or improve the survival rate after CABG, we hypothesized that epicardial repositioning of the PPM adjunct with mitral annular reduction would improve leaflet coaptation assessed by reduction of MR. We aimed to show how septolateral mitral annular reduction, overall papillary muscle tethering distances, 3D PPM geometry, papillary muscle tethering angle, and different methods of treatment influenced MR reduction. This study would demonstrate the feasibility of an epicardial correction to study geometric changes after mitral annular reduction alone compared with PPM repositioning adjunct with mitral annulus reduction in a pulsatile ex vivo heart model of FIMR.

\section{MATERIALS AND METHODS}

The ex vivo hearts preparation: Fresh ovine hearts from 36 adult Dorsett sheep $(50 \pm 7 \mathrm{~kg})$ with no cardiac abnormalities were collected and stored at $4^{\circ} \mathrm{C}$. Experimentation was done within 24 hours. The hearts were prepared and MR was induced by annular dilatation and PPMs displacement as described in previous study from Monnet et al [Monnet 2013]. An incision was made around the PPM without damaging the chordae to allow displacement of the PPM. The PPM remained intact with a $2 \mathrm{~cm}$ strip of myocardium at the apical $L V$ wall. The $L V$ wall around the PPM was sutured closed with a $3 \mathrm{~cm}$ diaphragmatic patch; this creates a focal dilation of the LV wall and cause outward displacement of the PPM. Kradangnga et al [Kradangnga 2018] studied the ex vivo pulsatile heart model of functional mitral regurgitation and showed that the 3 $\mathrm{cm}$ crescent-shaped diaphragmatic patch size placement induced the highest heart models with MR and also produced the highest MR volume. From this data, the $3 \mathrm{~cm}$ patch was used to increase the length of the $L V$ wall at the level of the PPM.

Studies have shown that annular dilatation in the septolateral direction along with papillary muscle displacement can produce MR [Green 1999]. In our study, we induced MR by both displacing the PPM away from the anterior mitral leaflet and increasing the septo-lateral annular dimension, mimicking LV remodeling and mitral annular dilatation in FIMR patients [Jensen 2010; Raman 2011; van Garsse 2012] The left atrium was incised to access the mitral annulus. The septo-lateral annular dimension was measured before inducing annular dilatation. The annular dilatation was achieved by mechanical stretch [Siefert 2012], without damaging the leaflet and chordae, in the septo-lateral direction until the diameter increased approximately 30 percent of the anterior mitral leaflet height [Khabbaz 2013; Siefert 2013; Tibayan 2003].

Six intracardiac $2 \mathrm{~mm}$ piezo-electric crystals $(2 \mathrm{~mm}$ round piezo-electric crystals, Sonometrics, London, Canada) were sutured to 6 mitral apparatus landmarks as illustrated in the Kradangnga et al study [Kradangnga 2018]. Nine crystal pairs were of interest and used for the LVV calculation and analysis of the annular and papillary muscles geometry (Table 1). Regional wall motion and distances between crystal pairs were measured continuously with a sonometric data acquisition system (SonoSoft, Sonometrics Corp., London, Canada). Three-dimensional (3D) coordination of each piezo-electric crystal was recorded at $200 \mathrm{~Hz}$ with simultaneous measurement of LVP [Gorman 1996; Jensen 2008].

Two suture markers were placed on the epicardium to locate APM and PPM bases. After piezo-electric crystals placement, the left atrium and diaphragmatic patch were sutured closed. The heart was mounted on a pulsatile pressurization system (Bio-console 520D Centrifugal Blood Pump, Bio Medicus, Minnesota), which generated pulsatile flow. We used the same setting of the pulsatile pressurization system as stated in a previous study [Kradangnga 2018]. The LVPmax was maintained at $120 \pm 5 \mathrm{mmHg}$. There was an aortic outflow valve that could be adjusted to decrease or increase outflow resistance until LVPmax reached desire value. Pulsatile flow was set at 4 liters per minute, 40 beats per minute with 
Table 3. Tethered Distances, 3D Geometry and Papillary Muscles Tethered Angles

\begin{tabular}{|c|c|c|c|c|c|}
\hline SL Annulus & $26.36[20.84,35.75]$ & $22.59 *[18.5,28.67]$ & $26.5[23.76,30.45]$ & $24.7 *[23.77,28.6]$ & .19 \\
\hline Fibrosa-APM tip (mm) & $45.47[40.8,50.19]$ & $45.73[43.25,52.8]$ & $45.3[40.3,51.1]$ & $48.75 *[42.83,51.1]$ & .23 \\
\hline Lateral annulus-APM tip $(\mathrm{mm})$ & $36.0[32.5,40.34]$ & $35.17[32.85,37.85]$ & $36.0[34.2,38.35]$ & $38.3[35.64,39.73]$ & .07 \\
\hline Lateral annulus-PPM tip (mm) & $33.55[30.76,37.19]$ & $33.7[31.06,36.9]$ & $32.72[29.8,34.7]$ & $31.4[28.2,33.25]$ & .11 \\
\hline Interpapillary muscle & $30.3[27.0,32.4]$ & $28.33[26.7,31.48]$ & $28.5[25.0,34.3]$ & $28.1 *[23.9,30.75]$ & .32 \\
\hline \multicolumn{6}{|l|}{ 3-Dimension of tethered distance } \\
\hline PPM Lateral (mm) & $47.93[39.07,55.43]$ & $47.49[40.68,55.45]$ & $44.37[29.13,55.71]$ & $43.88[36.86,52.88]$ & .10 \\
\hline APM Apical (mm) & $26.74[22.68,32.93]$ & $27.01[22.59,30.89]$ & $15.36[5.51,27.45]$ & $26.29[9.91,30.51]$ & .32 \\
\hline APM Anterior (mm) & $11.31[0.49,22.27]$ & $2.73[-7.31,18.25]$ & $7.95[2.02,12.31]$ & $8.9[-2.48,16.20]$ & .31 \\
\hline \multicolumn{6}{|l|}{ Tethered angles PPM tip } \\
\hline $\mathrm{F} \alpha\left(^{\circ}\right)$ & $33.01[24.67,44.04]$ & $29.11[16.15,39.22]$ & $26.24[15.51,29.17]$ & $27.48[24.06,40.55]$ & .04 \\
\hline $\mathrm{F} \beta\left(^{\circ}\right)$ & $111.44[101.68,132.96]$ & $132.10[111.71,146.05]$ & $124.08[83.83,133.8]$ & $129.48[114.5,133.39]$ & .16 \\
\hline $\mathrm{A} \alpha\left(^{\circ}\right)$ & $6.17[3.0,10.75]$ & $7.34[1.67,11.79]$ & $2.22[1.38,22.42]$ & $5.47[2.95,7.83]$ & .08 \\
\hline$A \beta\left(^{\circ}\right)$ & $154.26[138.71,173.74]$ & $164.11[149.15,175.94]$ & $167.22[73.25,175.58]$ & $167.65[153.59,174.27]$ & .08 \\
\hline \multicolumn{6}{|l|}{ APM tip } \\
\hline
\end{tabular}

Values are in medians and interquartile ranges. *Denotes $P<.05$ versus Baseline. $\nmid$ Relative change (\%) after epicardial device placement in MA+PPM versus MA group. APM, anterior papillary muscle; PPM, posterior papillary muscle; SL, septo-lateral; $F \alpha$, alpha angle in the frontal plane; $F \beta$, beta angle in the frontal plane; $A \alpha$, alpha angle in the annular plane; $A \beta$, beta angle in the annular plane.

the flow traveling from the LV apex into the aorta. If MR occurred, the MR flow will be collected and measured from the pulmonary vein cannula. A high-fidelity pressure catheter (Millar, Inc., Houston, Texas) was inserted into the LV in a retrograde fashion via the aorta. It was used for LVP measurement throughout the experiment.

The hearts randomly were assigned into 2 groups, depending on the method to lower or eliminated MR; they were the septo-lateral mitral annular reduction (MA) group and PPM repositioning adjunct with septo-lateral mitral annular reduction (MA+PPM) group.

Epicardial correction: In this study, we adapted an epicardial apparatus to alter PMs positions without changing annular dimension. The epicardial apparatus in our study consisted of 4 main parts, 2 cross bars, and 2 papillary muscles vertical bars (Figure 1A). The vertical bars had a retractable pushing pad that was placed on the epicardium at the level of the base of the APM and PPM. In this ex vivo study, we only adjust the retractable pushing pad at the PPM level. This pad was placed on the epicardium and aimed to push the PPM in a baso-medial direction toward the septal saddle horn of the annulus. Once the PPM vertical bar was placed at the desired position, the proximal of the vertical bar was secured with two mattress sutures apical to the atrioventricular groove and the distal part was secured at the apex. Care was taken while placing the apparatus to avoid coronary vessels. The first cross bar was used to connect the distal portion of the vertical bars. The second cross bar was connected between the proximal portions (Figure 1B). The length of the proximal cross bar could be adjusted to reduce the annular septo-lateral dimension.

After application of epicardial apparatus, the ex vivo heart was observed whether MR occurred or not. In the MA group, 


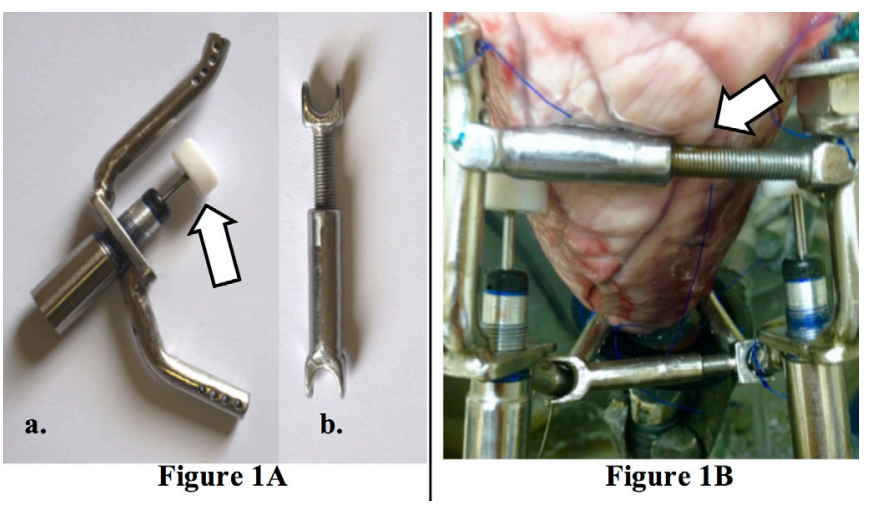

Figure 1. The epicardial device used for mitral annular dimension reduction and PPM repositioning. (1A) Papillary muscles vertical bar (a) and the cross bar (b). The white arrow indicates retractable pushing pad. (1B) Mounted epicardial device on the LV. The white arrow indicates the proximal cross bar.

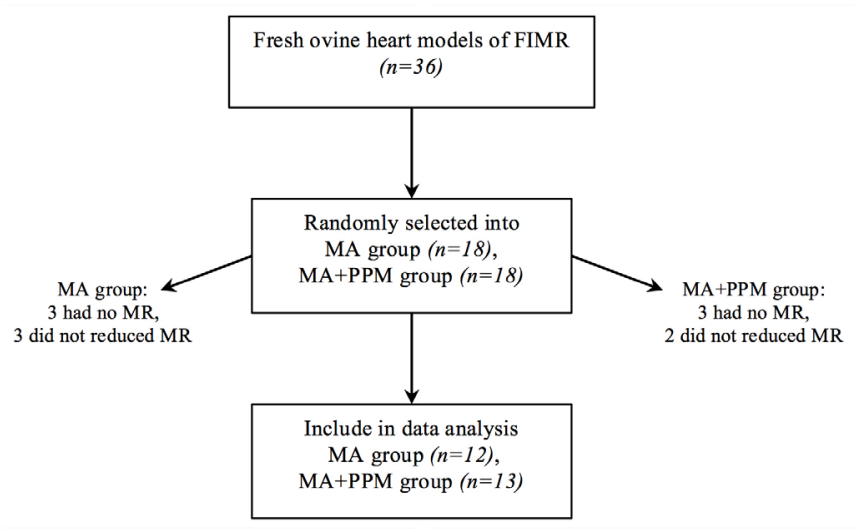

Figure 2. Flow chart of the data included in the analysis.

the proximal cross bar length was only adjusted until the maximum reduction, trace or no $M R$ was achieved. In the $\mathrm{MA}+\mathrm{PPM}$ group, the proximal cross bar length was adjusted until the maximum reduction of MR occurred then the vertical bar pushing pad at the level of PPM was adjusted to gain further maximum MR reduction. Hearts that failed to reproduce $M R$ at baseline or failed to reduced $M R$ after epicardial apparatus placement were excluded from the study (Figure 2).

\section{Data acquisition: Geometry analysis}

Data of the crystal pair distances were collected from each treatment group. Annular and papillary muscles geometry were analyzed at $\mathrm{LVP}_{\max }$, which represents the systolic phase when mitral valve closure occurs. Distances between crystal pairs were averaged over 10 pump cycles. Baseline data (Control) for each observation was collected before applying the LV epicardial contouring system and were used as its own control.

Three-dimensional assessments of the papillary muscles tip displacements were evaluated using Cartesian planes at $\mathrm{LVP}_{\max }$. The 3D planes were defined by the best-fitted plane, using four piezo-electric reference crystals (Crystal 1, 2, 3,
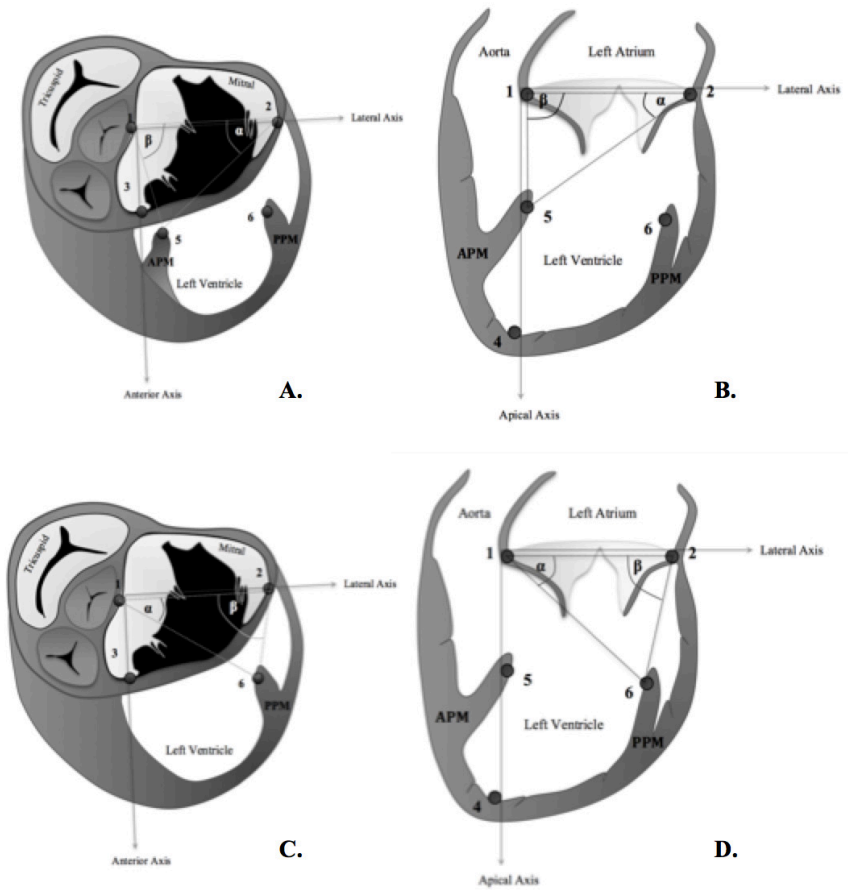

Figure 3. Illustrates the APM and PPM tethered angles in the annular plane and frontal plane. The numbers marked the landmarks of the crystal. The angles were marked as $\alpha$ and $\beta$. Crystal 1, 2, 3 and 4 were used as reference to construct Cartesian planes. (A, C) Oblique annular plane at the level of the mitral annulus. (B, D) Frontal plane of the left side of the heart.

and 4). Data from these crystals were analyzed with SonoXYZ software (Sonosoft, Sonometrics, London, Canada) to calculate the Cartesian coordinates throughout the time course of the experiment. We used the same method as in the previous chapter to construct the annular plane ( $\mathrm{XZ}$ planar), frontal plane $(\mathrm{XY})$, and antero-posterior plane (YZ planar). The $\mathrm{XZ}$, $\mathrm{XY}$, and XZ Cartesian coordinate planes were perpendicular to one another. The crystal at the level of the anterior annulus (C1) was used as the origin of 3 perpendicular vector axes [Richards 2009]. The positive lateral axis $(+\mathrm{X})$ passed through the mid-lateral annular crystal (C2). The positive apical axis $(+\mathrm{Y})$, perpendicular with the lateral axis, was directed toward the apical crystal (C4). The positive anterior axis $(+Z)$, perpendicular with the lateral axis and apical axis, was calculated with SonoXYZ software directed toward the annular anterior commissure crystal (C3). The APM and PPM positions at $\mathrm{LVP}_{\text {max }}$ were determined into their septo-lateral (X), anteroposterior $(\mathrm{Z})$, and baso-apical $(\mathrm{Y})$ components.

The tethered degree of angle ( $\alpha$ or $\beta$ ) of the AMP and PPM tips, reflecting restriction from displaced PPM and annular dilation, were evaluated. The angles were calculated in the annular and frontal planes as stated in Figure 3. The $\alpha$ angle of the PPM was defined as the angle across the PPM tip. It was formed by C1-C2 segment and C1-C6 segment. The $\alpha$ angle of the APM was defined as the angle across the APM tip, formed by C1-C2 segment and C2-C5 segment. The $\beta$ angle of the PPM was form by C1-C2 segment and 

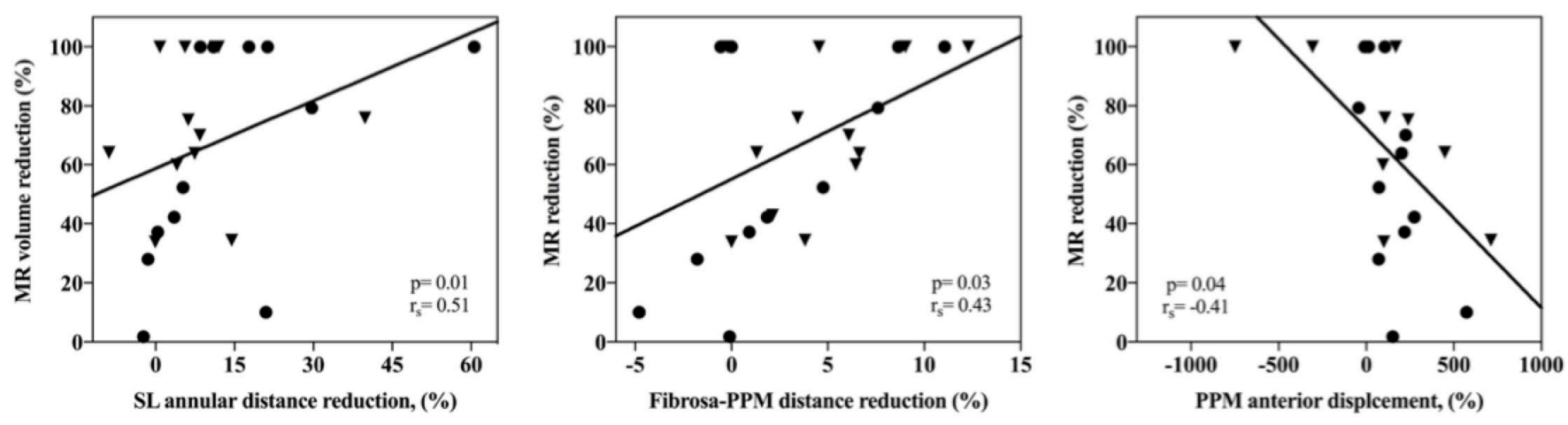

Figure 4. Scatter plots and Spearman correlation coefficient of MR volume reduction and left, SL annular distance reduction; middle, fibrosa-PPM distance reduction; right, PPM anterior displacement in percentages. In each subplot, $r_{s}$ is Spearman correlation coefficient. Scatter plots from MA group.

C2-C6 segment. The $\beta$ angle of the APM was form by C1-C2 segment and C1-C5 segment. The tethered angles were compared to baseline and between the groups.

Quantification of MR, left ventricular volume and mitral annular area analysis: MR stroke volume (milliliters per beat) was quantified by direct measurements of MR volume that exceed from the pulmonary vein port. MR stroke volume above 5 milliliters per beat was considered significant. MR stroke volume was measured and averaged over 10 pump cycles. The percentage of MR reduction from baseline was calculated. In this study, we modified quantification of MR by the measurements of the MR stroke volume from the AHA/ ACC guidelines [Members, 2006 Writing Committee 2008] and Zoghbi et al study [Zoghbi 2003]. The MR severity was graded on a scale of 0 to 4 , in which $0=$ no to trace $M R$ (MR $<5 \mathrm{~mL} /$ beat), $1=$ mild (MR 5-20 mL/beat), $2=$ moderate (MR 20-30 mL/beat), 3 = moderate to severe (MR 30-40 mL/ beat), and 4 = severe (MR > $40 \mathrm{~mL} /$ beat.

The LVPmax resembled the systolic phase in the normal cardiac cycle when the mitral valve was closed. MR should occur in this phase if there were mal-coaptation of the leaflets. Left ventricular volume was obtained and calculated by sonometric data acquisition system (CardioSoft, Sonometrics Corp., London, Canada) at $\mathrm{LVP}_{\max }$. The 'Ellipsoid model' equation was used for the LVV calculation. This model used three axes (Major axis, minor axis I, and minor axis II). The distance between Crystal 1 and 2 was used as minor axis I. The distance between the anterior commissure of the annulus and the apex was used as major axis. The minor axis II was the axis between the tips of APM-PMM.

$$
\mathrm{LVV}=\frac{\pi * \text { major axis } * \text { minor axis } * \text { minor axis } I I}{6}
$$

Mitral annular area (MAA) before and after treatment was compared. MAA was calculated with the following equation: MAA $=\pi\left(r_{1}\right)\left(r_{2}\right) / 4 ; r_{1}$ is the diameter of the septo-lateral annulus and $r_{2}$ is the inter-commissure diameter. After the experiment was completed, the LV was opened for exploration to confirm no damage was made to the mitral apparatus.
Data were analyzed using SAS version 9.4 (SAS University Edition, Institute Inc., Cary, North Carolina) with significance at $P<.05$ or stated values. Relative percentage change from baseline was calculated for each variable. Shapiro-Wilk test for normality distribution was tested before analysis. Data with numeric results were presented as median and interquartile range (median [Q1, Q3]), since normality of the data was not achieved. Wilcoxon Sign-ranked test was used for comparison of the variables at baseline and after treatment methods. Percentage relative changes between the MA and $\mathrm{MA}+\mathrm{PPM}$ groups were compared using a Wilcoxon Ranksum test. Spearman correlation coefficient was used to present association between MR reduction and geometric data variables. Variables that were associated with MR reduction will be further analyzed.

\section{RESULTS}

Left ventricular volume, MAA, and MR are reported in Table 2. Mitral annular area significantly decreased from baseline in the MA group $(P=.004)$. LVV significantly decreased in the MA $(P=.02)$ and MA+PPM $(P=.01)$ groups when compared with its own baseline. MR severity grade significantly decreased from baseline in both the MA $(P=.03)$ and $\mathrm{MA}+\mathrm{PPM}$ groups $(P=.02)$. However, the $\mathrm{MA}+\mathrm{PPM}$ group did not significantly have $M R$ grade and MR reduction lower than MA group (Table 2).

The overall tethered distances and 3D displacement of the papillary muscles were reported in Table 3 . The septo-lateral mitral annular distance significantly decreased after applying both methods (MA group $P=.005 ; \mathrm{MA}+\mathrm{PPM}$ group $P=$ $.05)$. The tethered distances of the fibrosa to PPM tip and the interpapillary muscles significantly decreased from baseline in the MA+PPM group $(P=.02$ and $P=.047$, respectively). The tethered distances of the fibrosa to APM tip significantly increased from baseline in the MA+PPM group $(P=.002)$. However, there were no statistically significant differences of the septo-lateral mitral annular distances and geometry of the papillary muscles tethered distances found between the 2 groups. 
The tethered angles of the papillary muscles referred to the septo-lateral mitral annular segment are reported in Table 3 . The tethering $\alpha$ angle of the APM in the frontal plane significantly increased from baseline in the MA+PPM group $(P=.027)$. Furthermore, the MA+PPM group had a larger APM and PPM $\alpha$ angle in the frontal plane compared with the MA group after reducing the MR $(P=.04)$. There were no statistically significant changes in tethering angles found in the MA group compared with baseline.

When including all hearts with MR reduction after applying treatment methods, the percentage of MR reduction correlated with the percentage decrease of septo-lateral mitral annular distance $\left(r_{s}=0.51, P=.01\right)$, percentage decrease of fibrosa-PPM distance $\left(r_{s}=0.43, P=.03\right)$, and percentage increase of the PPM anterior displacement $\left(r_{s}=-0.41\right.$, $P=.04$ ) (Figure 4).

\section{DISCUSSION}

Chronic FIMR, typically after posterior-lateral myocardial infarction, caused left ventricle (LV) remodeling [Kumanohoso 2003]. Although LV remodeling displaced both PMs (even the one which is not involved by the infarction) as the entire LV dilated; the APM displacement was minimal compared with the PPM. This event mainly caused asymmetrical PPM displacement at the LV wall where ischemia occurred [Jensen 2010; Raman 2011]. FIMR patients have restricted leaflet closure due to tethering force influenced by the displaced PMs and concurrent dilated annulus. Monnet and colleagues have shown that annular reduction in septolateral dimension was more significant in reducing MR [Monnet 2013]. Although the concept of ring annuloplasty was to bring the septo-lateral annular dimension closer together so that the mitral leaflets could completely form coaptation, surgical annuloplasty also could potentially increase PML tethering by relatively displacing the PPM outside the mitral annular ring [Green 1999; Hung 2004]. Despite excellent long-term results, recurrent FIMR and ongoing LV remodeling could be found post-annuloplasty [Gillinov 2001].

The concept of reducing PMs tethering by combining subvalvular techniques to normal reduction annuloplasty was adapted by many researchers. These techniques include PMs relocation, trans-ventricular suture technique, PMs approximation (PMA), chordal cutting and trans-ventricular devices. These techniques aimed to eliminate MR by reducing the tethered force on the mitral leaflets. Treatment of moderate to severe FIMR with annuloplasty alone seemed uncertain and the decision to add subvalvular technique depends on each individual case. Szymanski and colleagues [van Garsse 2012] showed that in chronic FIMR sheep models secondorder mitral chordal cutting improved annuloplasty by reducing papillary muscle tethering. Subvalvular techniques that reposition the whole body of PMs could help avoid the stress on the PMs tips and some part of the annulus such as PPM approximation [Siefert 2012].

The goal of our study was to evaluate a potential synergistic effect of the association of septo-lateral annular reduction with PPM repositioning. PPM repositioning via epicardial pushing pad at the level of the PPM should give information on how manipulating a single PM could lower MR. This also introduced a further step in developing epicardial device, lessening the need of open heart surgery. Our study showed that the combined MA+PPM using the epicardial adjustable pads directly repositioned the outwardly displaced PPM and reduced the MR without further compromising the MAA.

The PPM in chronic FIMR was displaced more laterally in chronic FIMR sheep models [Zoghbi 2003]. They also found that the PML margin was displaced apically in chronic FIMR. Therefore to reduce MR, reversal of these geometric changes should be made. Although we did not see significant changes in the 3D PPM geometry after applying the MA+PPM group, our study revealed a decrease of the fibrosa-PPM distance and the interpapillary muscle distance. This could indicate the baso-medial repositioning of the PPM tip. The interpapillary muscle distance was decreased after adjusting the PPM pushing pad to reduce/eliminate MR. Reduction of the interpapillary muscles distance should reduce the tethering force on the leaflets, especially improving the posterior leaflet mobility. Distance between the papillary muscles was a predictor for FIMR severity. Studies in both animal models and human patients have shown that increased interpapillary muscle distance was associated with the severity of FIMR. Jensen and colleagues [Jensen 2010] used porcine FIMR models and found that the interpapillary muscle distance was the only independent predictor for FIMR severity. A study in patients with chronic FIMR post CABG and reduction ring annuloplasty also showed that the pre-operative interpapillary muscle distance was associated with anterior mitral leaflet tethering, which was associated with recurrent FIMR [Zoghbi 2003]. Combined PPM reposition with annular reduction lowered PPM tethering, which reduced tethering force on the leaflets, especially improving the posterior leaflet mobility. In our study, the tethered length and angles of the PMs were measured instead of directly measuring from the mitral leaflets. We aim to demonstrate how manipulating the PPM via the epicardial can change the PPM geometry similar to extra-cardiac subvalvular techniques for FIMR treatment. These extra-cardiac subvalvular techniques have been established to avoid CPB surgery, aiming to reduce the LV dilatation and interpapillary distance [Acker 2006; Acker 2011; Richards 2009; Siefert 2012].

Tethered angle of the PMs was studied to give us more information on the changes of the biomechanical mechanisms in heart models that reduced/eliminated MR after treatment. Adjustments of the epicardial pushing pad at the level of the PPM altered both mitral leaflets, since the PPM suspend both mitral leaflets. In our recent study, we found that the PPM frontal plane $\alpha$ angle decreased 10 degrees in hearts with MR. Therefore, increasing the PPM frontal plane $\alpha$ angle should reduce MR. In this study, the tethered frontal plane $\alpha$ angle of the PPM in the combined treatment group was relatively increased from baseline $\left(15.83 \pm 33.34^{\circ}\right)$ compared with the MA alone group. This finding along with the decrease of the interpapillary muscles distance suggested that the PPM was 
repositioned inward and toward the septal annulus by the epicardial pushing pad. Our results demonstrated that combined PPM repositioning via the epicardial apparatus associated with septo-lateral annular reduction in ovine heart models of FIMR setting improves MR as a result of improved coaptation and reduced interpapillary muscle distance.

Although the ex vivo pulsatile heart model of FIMR produces annular dilatation and PPM geometric changes similar to previous studies of FIMR patients [Gorman 2004; Tibayan 2003], limitations can be found in this study. The primary limitation of this study is that the heart model does not have functional myocardium. LVP and MR were generated by the pressurization of the dynamic pump system. Physiological function of the $\mathrm{LV}$ after device placement cannot be assessed such as the ejection fraction, LV elasticity, and evaluation of the myocardial blood flow. Further concerns for myocardial blood flow alteration are focal ischemia of the $\mathrm{LV}$ myocardium initiate by the contact pressure from the pushing pads. Similar studies of epicardial device that apply pressure on the myocardial wall have not demonstrated focal ischemia from device placement in animal models [Inoue 2004; Kashem 2013].

From this study, we did not know exactly which tethered leaflet resolved after treatment since the PPM that was repositioned had chordae suspended to both leaflets. Leaflet tethering angles using $3 \mathrm{D}$ echocardiography with a standard reference system would assist further study on the leaflet 3D geometry. Another limitation of this study is the lack of validation on aggressive annular reduction. In our study, we only adjusted the proximal cross bar of the epicardial device to reduce the annular dimension until the maximum reduction of MR, trace or no MR was achieved. Although, we found mitral stenosis in one observation with total MR elimination after MA treatment, this was excluded from the study. Aggressive annular reduction without further causing mitral stenosis along with PPM repositioning may have improved reduction of MR.

\section{REFERENCES}

Acker MA, Bolling S, Shemin R, et al. 2006. Mitral valve surgery in heart failure: Insights from the Acorn Clinical Trial. J Thorac Cardiovasc Surg 132:568-577.e4.

Acker MA, Jessup M, Bolling SF, et al. 2011. Mitral valve repair in heart failure: Five-year follow-up from the mitral valve replacement stratum of the Acorn randomized trial. J Thorac Cardiovasc Surg 142:569-574.e1.

Badiwala MV, Verma S, Rao V. 2009. Surgical Management of Ischemic Mitral Regurgitation. Circulation 120:1287-1293.

Bolling SF. 2001. Mitral valve reconstruction in the patient with heart failure. Heart Fail Rev 6:177-85.

Bursi F, Enriquez-Sarano M, Nkomo VT, et al. 2005. Heart Failure and Death After Myocardial Infarction in the Community The Emerging Role of Mitral Regurgitation. Circulation 111:295-301.

Calafiore AM, Gallina S, Di Mauro M, et al. 2001. Mitral valve procedure in dilated cardiomyopathy: repair or replacement? Ann Thorac Surg 71:1146-1152.

Daneshmand MA, Milano CA, Rankin JS, et al. 2009. Mitral Valve Repair for Degenerative Disease: A 20-Year Experience. Ann Thorac
Surg 88:1828-1837.

Fattouch K, Sampognaro, R, Speziale G, et al. 2010. Impact of Moderate Ischemic Mitral Regurgitation After Isolated Coronary Artery Bypass Grafting. Ann Thorac Surg 90, 1187-1194.

Gillinov AM, Wierup PN, Blackstone EH, et al. 2001. Is repair preferable to replacement for ischemic mitral regurgitation? J Thorac Cardiovasc Surg 122:1125-1141.

Gorman III JH, Gupta KB, Streicher JT, et al. 1996. Dynamic threedimensional imaging of the mitral valve and left ventricle by rapid sonomicrometry array localization. J Thorac Cardiovasc Surg 112, 712-724.

Gorman III JH, Jackson BM, Enomoto Y, Gorman RC. 2004. The effect of regional ischemia on mitral valve annular saddle shape. Ann Thorac Surg 77:544-548.

Green GR, Dagum P, Glasson JR, et al. 1999. Mitral Annular Dilatation and Papillary Muscle Dislocation Without Mitral Regurgitation in Sheep. Circulation 100:II95-II102.

Green GR, Dagum P, Glasson JR, et al. 1999. Restricted posterior leaflet motion after mitral ring annuloplasty. Ann Thorac Surg 68:2100-2106.

Hung J, Papakostas L, Tahta SA, et al. 2004. Mechanism of Recurrent Ischemic Mitral Regurgitation After Annuloplasty Continued LV Remodeling as a Moving Target. Circulation 110:II85-II90.

Inoue M, McCarthy PM, Popović ZB, et al. 2004. The Coapsys device to treat functional mitral regurgitation: in vivo long-term canine study. J Thorac Cardiovasc Surg 127:1068-1077.

Jensen H, Jensen MO, Ringgaard S, et al. 2008. Geometric determinants of chronic functional ischemic mitral regurgitation: insights from threedimensional cardiac magnetic resonance imaging. J Heart Valve Dis 17:16-22.

Jensen H, Jensen MO, Smerup MH, et al. 2010. Three-dimensional assessment of papillary muscle displacement in a porcine model of ischemic mitral regurgitation. J Thorac Cardiovasc Surg 140:1312-1318.

Jensen H, Jensen MO, Nielsen SL. 2015. Surgical Treatment of Functional Ischemic Mitral Regurgitation. J Heart Valve Dis 24:30-42.

Kashem A, Hassan S, Crabbe DL, Melvin DB, Santamore WP, Left ventricular reshaping: Effects on the pressure-volume relationship. 2013. J Thorac Cardiovasc Surg 125:391-9.

Khabbaz KR, Mahmood F, Shakil O. 2013. Dynamic 3-Dimensional Echo-cardiographic Assessment of Mitral Annular Geometry in Patients With Functional Mitral Regurgitation. Ann Thorac Surg 95:105-110.

Kradangnga K. and Monnet E. 2018. Left ventricular dilatation and posterior papillary muscle displacement in an ex vivo pulsatile model of functional mitral regurgitation. Heart Surg Forum 21(4):275-280.

Kumanohoso T, Otsuji Y, Yoshifuku S, et al. 2003. Mechanism of higher incidence of ischemic mitral regurgitation in patients with inferior myocardial infarction: Quantitative analysis of left ventricular and mitral valve geometry in 103 patients with prior myocardial infarction. J Thorac Cardiovasc Surg 125:135-143.

McGee Jr EC, Gillinov AM, Blackstone EH, et al. 2004. Recurrent mitral regurgitation after annuloplasty for functional ischemic mitral regurgitation. J Thorac Cardiovasc Surg 128:916-924.

Members, 2006 Writing Committee, Bonow RO, Carabello BA, Chatterjee K, et al. 2008. 2008 Focused Update Incorporated Into the ACC/AHA 2006 Guidelines for the Management of Patients With Valvular Heart Disease A Report of the American College of Cardiology/American Heart Association Task Force on Practice Guidelines 
(Writing Committee to Revise the 1998 Guidelines for the Management of Patients With Valvular Heart Disease): Endorsed by the Society of Cardiovascular Anesthesiologists, Society for Cardiovascular Angiography and Interventions, and Society of Thoracic Surgeons. Circulation 118:e523-e661.

Members AF, Vahanian A, Alfieri O, et al. 2012. Guidelines on the management of valvular heart disease (version 2012). Eur Heart J $33: 2451-2496$.

Members WC, Hillis LD, et al. 2011. 2011 ACCF/AHA Guideline for Coronary Artery Bypass Graft Surgery A Report of the American College of Cardiology Foundation/American Heart Association Task Force on Practice Guidelines. Circulation 124:e652-e735.

Monnet E, Pouching K. 2013. An Ex Vivo Model of Left Ventricular Dilation and Functional Mitral Regurgitation to Facilitate the Development of Surgical Techniques. Heart Surg Forum 16:329-335.

Nishimura RA, Otto CM, Bonow RO, et al. 2017. 2017 AHA/ACC Focused Update of the 2014 AHA/ACC Guideline for the Management of Patients With Valvular Heart Disease: A Report of the American College of Cardiology/American Heart Association Task Force on Clinical Practice Guidelines. Circulation.

Otsuji Y, Levine RA, Takeuchi M, Sakata R, Tei C. 2008. Mechanism of ischemic mitral regurgitation. J Cardiol 51:145-156.

Raman J, Jagannathan R, Chandrashekar P, Sugeng L. 2011. Can We
Repair the Mitral Valve from Outside the Heart? A Novel Extra-Cardiac Approach to Functional Mitral Regurgitation. Heart Lung Circ 20:157-162.

Richards AL, Cook RC, Bolotin G, Buckner GD. 2009. A Dynamic Heart System to Facilitate the Development of Mitral Valve Repair Techniques. Ann Biomed Eng 37:651-660.

Siefert AW, Jimenez JH, Koomalsingh KJ, et al. 2012. Dynamic Assessment of Mitral Annular Force Profile in an Ovine Model. Ann Thorac Surg 94:59-65.

Siefert AW, Rabbah JPM, Koomalsingh KJ, et al. 2013. In Vitro Mitral Valve Simulator Mimics Systolic Valvular Function of Chronic Ischemic Mitral Regurgitation Ovine Model. Ann Thorac Surg 95:825-830.

Tibayan FA, Rodriguez F, Zasio MK, et al. 2003. Geometric Distortions of the Mitral Valvular-Ventricular Complex in Chronic Ischemic Mitral Regurgitation. Circulation 108:II116-II121.

van Garsse L, Gelsomino S, Luca F, et al. 2012. Importance of anterior leaflet tethering in predicting recurrence of ischemic mitral regurgitation after restrictive annuloplasty. J Thorac Cardiovasc Surg 143(4):S54-9.

Zoghbi WA, Enriquez-Sarano M, Foster E, et al. 2003. Recommendations for evaluation of the severity of native valvular regurgitation with two-dimensional and doppler echocardiography. J Am Soc Echocardiogr $16: 777-802$. 